\title{
Membership grade mining of mutually inverse fuzzy implication propositions
}

\author{
Xunwei Zhou ${ }^{1}$
}

Received: 24 March 2016/Accepted: 27 July 2016/Published online: 6 August 2016

(C) Springer International Publishing Switzerland 2016

\begin{abstract}
Mutually inverse implication proposed by the author inherits from and makes improvement to material implication. Mutually inverse fuzzy implication proposed by the author combines mutually inverse implication and fuzzy logic. The algorithm of membership grade mining of mutually inverse fuzzy implication propositions is given, its computational complexity is almost linear.
\end{abstract}

Keywords Material implication - Mutually inverse implication · Fuzzy logic · Mutually inverse fuzzy implication $\cdot$ Data mining

\section{Introduction}

The truth table for material implication in classical logic (Hamilton 1978) correctly reflects the establishment of sufficient but not necessary condition. The truth table for equivalence correctly reflects the establishment of sufficient and necessary condition. Mutually inverse implication in mutually-inversistic logic (Zhou 2013) proposed by the author inherits the valuable part of material implication, and makes several improvements. The inductive composition truth table for mutually inverse implication combines the truth tables for material implication and equivalence, reflecting the establishment of sufficient condition. In classical logic, after people have established a material implication proposition using the truth table for material implication, and people want to use it as the major premise

Xunwei Zhou

zhouxunwei@263.net

1 Institute of Information Technology, Beijing Union University, Beijing, China to make hypothetical inference, people still use the same truth table to do it. While in mutually-inversistic logic, after we have established a mutually inverse implication proposition using inductive composition truth table, and we want to use it as the major premise to make hypothetical inference, we use its inverse functions to do so. In material implication there are implicational paradoxes: If the antecedent is false or the consequent is true, then the antecedent implies the consequent; there can be no nexus of contents between the antecedent and the consequent. Mutually inverse implication is free from implicational paradoxes by requiring that the antecedent is not permanently false, the consequent is not permanently true; and that the antecedent and the consequent share the same variable, the idea comes from relevance logic (Anderson and Jr 1975). In the predicate calculus of classical logic there are quantifiers. While in the predicate calculus of mutually-inversistic logic quantifiers are not introduced.

Mutually inverse fuzzy implication combines mutually inverse implication with fuzzy logic (Zadeh 1965). The mutually inverse fuzzy implication proposition studied in this paper is in the form of $\frac{w 1 a 1+w 2 a 2}{w 1+w 2} \leq_{\mathrm{f}}^{-1} c$, where $a_{1}$ and $a_{2}$ are the two antecedents, $w_{1}$ and $w_{2}$ are their weights, $\leq_{\mathrm{f}}^{-1}$ is mutually inverse fuzzy implication, $c$ is the consequent. This paper studies the mining of membership grade of the mutually inverse fuzzy implication proposition $\frac{w 1 a 1+w 2 a 2}{w 1+w 2} \leq{ }_{f}^{-1} c$ from those of the antecedents $a_{1}$ and $a_{2}$ and the consequent $c$. The mining is divided into two steps. The first step is for a few instances of $a_{1}, a_{2}$, and $c$, solves the simultaneous equations

$\left\{\begin{array}{l}w_{1} a_{11}+w_{2} a_{12}=c_{1} \\ w_{1} a_{21}+w_{2} a_{22}=c_{2}\end{array}\right.$

to determine the weights $w_{1}$ and $w_{2}$. The computational complexity of this step is polynomial. Although OWA 
(Yager 1988; Emrouznejad and Marra 2014) has nice properties, it is not applicable in this step. Because $a_{1}$ and $a_{2}$ range over a number of instances, they cannot be ordered, e.g., $a_{11}$ might be 0.6 and $a_{12}$ might be 0.9 while $a_{21}$ might be 0.8 and $a_{22}$ might be 0.7 . The second step is for the majority instances of $a_{1}, a_{2}$, and $c$, from their membership grades to mine that of $\frac{w 1 a 1+w 2 a 2}{w 1+w 2} \leq{ }_{\mathrm{f}}^{-1} c$. The computational complexity of this step is linear. Since the first step only concerns a few instances, the second step concerns the majority of instances, the overall computational complexity is nearly linear. The difference between the membership grade mining of the mutually inverse fuzzy implication proposition and the fuzzy association rule mining (Kuok et al. 1997) is that the former mines the membership grade, while the latter mines the support and the confidence.

The rest of this paper is organized as follows: in Sect. 2 material implication and equivalence are introduced, in Sect. 3 mutually inverse implication is introduced, in Sect. 4 fuzzy logic is introduced, in Sect. 5 mutually-inversistic fuzzy logic is introduced, in Sect. 6 membership grade mining of mutually inverse fuzzy implication propositions is introduced, in Sect. 7 mutually-inverse fuzzy logic and Zadeh's fuzzy logic are compared, in Sect. 8 the concluding remarks is given.

\section{Material implication and equivalence}

\subsection{Material implication}

Material implication $\rightarrow$ is defined in Table 1 .

Table 1 correctly reflects the establishment of sufficient but not necessary condition. Before $A \rightarrow B$ is established, the universe of discourse is shown in Fig. 1. The first, second, third, and fourth row of Table 1 correspond to the compartment I, II, III, and IV of Fig. 1, respectively.

Suppose $A$ is "it rains", $B$ is "the ground is wet", $A \rightarrow B$ is "if it rains, then the ground is wet". We take "if it rains, then ground is wet" as an example, to show the establishment of $A \rightarrow B$. "It does not rain and the ground is not wet" is true, this is described by the first row of Table 1 and the compartment I of Fig. 1 is not empty. "It does not rain, but the ground is wet" is true (e.g. sprayers spray water on the ground), this is described by the second row of Table 1 and the compartment II of Fig. 1 is not empty. "It

Table 1 Material implication $\rightarrow$

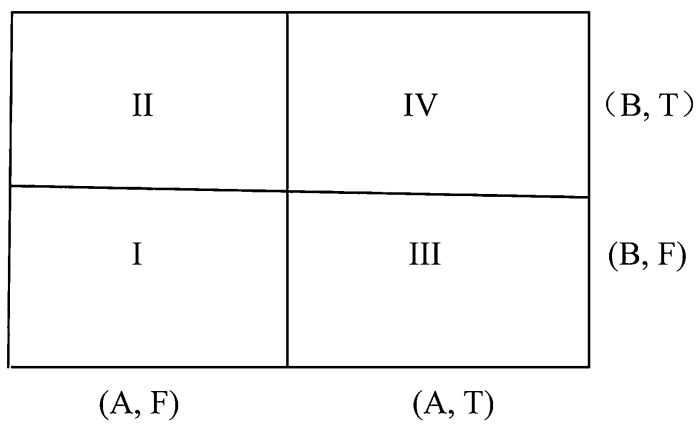

Fig. 1 Before $A \rightarrow B$ established

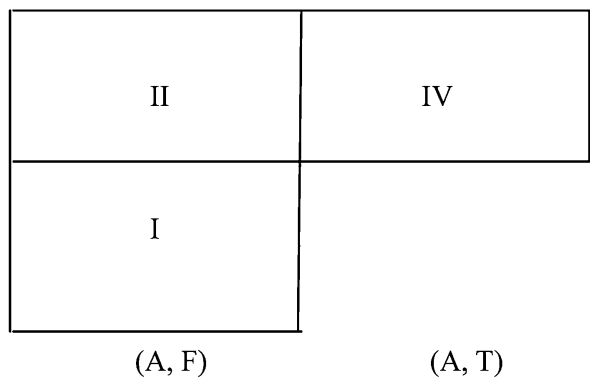

Eig. 2 After $\mathrm{A} \rightarrow \mathrm{B}$ is established

Fig. 2 After $A \rightarrow B$ established

Fig. 3 Another way of drawing Fig. 2

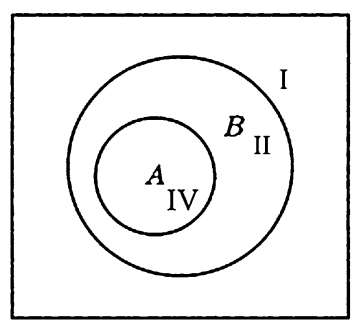

rains, and the ground is wet" is true, this is described by the fourth row of the Table 1 and compartment IV of Fig. 1 is not empty. "It rains but the ground is not wet" is false, this is described by the third row of Table 1 and the compartment III of Fig. 1 is empty. So from "it rains" and the "ground is wet", we establish "if it rains, then the ground is wet" using Table 1. After the establishment, we retain the non-empty compartments of Fig. 1, delete the empty compartment of Fig. 1, thus, Fig. 1 becomes Fig. 2. Figure 2 can be redrawn as Fig. 3

From Fig. 3 we see that when $A$ is true, $B$ is necessarily true; but when $B$ is true, $A$ may not be true. That is, Fig. 3 reflects that $A$ is a sufficient but not necessary condition of $B$.

\subsection{Equivalence}

The definition of equivalence $\leftrightarrow$ is shown in Table 2 .

Table 2 correctly reflects the establishment of sufficient and necessary condition. After $A \leftrightarrow B$ is established, we obtain Fig. 4. 
Table 2 Equivalence $\leftrightarrow$

\begin{tabular}{llll}
\hline$A$ & $B$ & । & $A \leftrightarrow B$ \\
\hline$F$ & $F$ & । & $T$ \\
$F$ & $T$ & । & $F$ \\
$T$ & $F$ & । & $F$ \\
$T$ & $T$ & । & $T$ \\
\hline
\end{tabular}

Fig. 4 After $A \leftrightarrow B$ is established

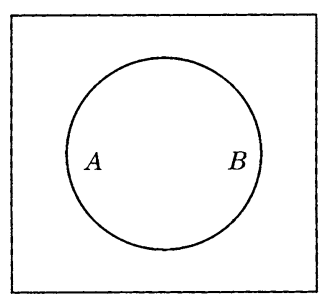

From Fig. 4 we see that when $A$ is true, $B$ is necessarily true; and when $B$ is true, $A$ is necessarily true. That is, Fig. 4 reflects that $A$ is a sufficient and necessary condition of $B$.

\section{Mutually inverse implication}

Mutually inverse implication $\leq^{-1}$ inherits the value of material implication and equivalence, and makes several improvements.

\subsection{Inductive composition truth table of $\leq^{-1}$}

The inductive composition truth table of $\leq^{-1}$ is shown in Table 3.

In Table 3, $n$ means "need not determine whether it is true or false". Table 3 combines Tables 1 and 2, reflecting the establishment of sufficient condition. Table 3 is obtained as follows: The common part of $A \rightarrow B$ and $A \leftrightarrow B$ is that in both propositions $A$ is a sufficient condition of $B$. The common part of Tables 1 and 2 is their first, third, and fourth row. Since $A \leq^{-1} B$ means that $A$ is a sufficient condition of $B$, the first, third, and fourth row of Table 3 are the same as those of Tables 1 and 2. The different part of $A \rightarrow B$ and $A \leftrightarrow B$ is that in $A \rightarrow B B$ is not a sufficient condition of $A$, while in $A \leftrightarrow B B$ is a sufficient condition of $A$. The different part of Tables 1 and 2 is their

Table 3 The inductive composition truth table of $\leq^{-1}$

\begin{tabular}{llll}
\hline$A$ & $B$ & । & $A \leq^{-1} B$ \\
\hline$F$ & $F$ & । & $T$ \\
$F$ & $T$ & । & $n$ \\
$T$ & $F$ & । & $F$ \\
$T$ & $T$ & । & $T$ \\
\hline
\end{tabular}

second row. Since $A \leq{ }^{-1} B$ does not care whether $B$ is a sufficient condition of $A$ or not, the second row of Table 3 is $n$.

\subsection{Decomposition truth tables of $\leq^{-1}$}

In classical logic, when people have established $A \rightarrow B$ using Table 1 and people want to use it as the major premise to make hypothetical inference, people still use Table 1 to do so. The affirmative expression of hypothetical inference is done as follows: Both $A \rightarrow B$ and $A$ being true is the fourth row of Table 1 , in which $B$ is true. The negative expression of hypothetical inference is done as follows: $A \rightarrow B$ being true and $B$ being false is the first row of Table 1 , in which $A$ is false.

This won't do. There is a fundamental principle in philosophy that human cognition is from the known to the unknown. There is a fundamental principle in mathematics that the evaluation of a function is from the arguments to the value, if we want to evaluate from the value to the argument, then we must use its inverse functions. To mathematize human cognition, we take the known as the argument, take the unknown as the value, thus the human cognition from the known to the unknown becomes the evaluation of a function from the arguments to the value. In Table $1, A$ and $B$ are the known, the arguments, $A \rightarrow B$ is the unknown, the value. So Table 1 can only be used to establish $A \rightarrow B$ from $A$ and $B$. After $A \rightarrow B$ has been established by Table 1 , and we want to use it as the major premise to make hypothetical inference, we should use the inverse functions of Table 1 to do so. Using Table 1 to make hypothetical inference is from the unknown to the known, from the value to the argument, violating the fundamental principles of philosophy and mathematics.

Following this clue, the generalized inverse functions of Table 3 are Tables 4 and 5 .

Table 4 Decomposition truth table one of $A \leq^{-1} B$

Table 5 Decomposition truth table two of $A \leq^{-1} B$

\begin{tabular}{llll}
\hline$A \leq^{-1} B$ & $A$ & । & $B$ \\
\hline$F$ & $F$ & । & $u$ \\
$F$ & $T$ & । & $u$ \\
$T$ & $F$ & । & $u$ \\
$T$ & $T$ & । & $T$ \\
\hline
\end{tabular}

\begin{tabular}{llll}
\hline$A \leq^{-1} B$ & $B$ & । & $A$ \\
\hline$F$ & $F$ & । & $u$ \\
$F$ & $T$ & । & $u$ \\
$T$ & $F$ & । & $F$ \\
$T$ & $T$ & । & $u$ \\
\hline
\end{tabular}


In Tables 4 and 5, $u$ means "unable to determine whether it is true or false". Tables 4 and 5 are established empirically. Take the second row of Table 4 as an example. Suppose $A$ is integer $(x), B$ is positive_number $(x)$, then the major premise $A \leq^{-1} B$ becomes integer $(x) \leq^{-1-}$ positive_number $(x)$ which is false (note that in mutuallyinversistic predicate calculus, quantifiers are not introduced, $x$ 's occurring on both sides of $\leq^{-1}$ are bound). And when the minor premise integer $(x)$ is true, we cannot determine the truth and falsity of the conclusion positive_number $(x)$. Because $x$ can be assigned 2, then the conclusion positive_number(2) is true; and $x$ can also be assigned -2 , then the conclusion positive_number $(-2)$ is false.

After we have established $A \leq^{-1} B$ by Table 3, it becomes known, becomes the argument. At this time, if we want to use it as the major premise to make hypothetical inference, then we use Tables 4 or 5 to do so, because in Tables 4 and $5, A \leq^{-1} B$ is the known, the argument. Using Tables 4 and 5 to make hypothetical inference does not violate the fundamental principles of philosophy and mathematics. Table 4 is the affirmative expression of hypothetical inference: if $A \leq{ }^{-1} B$ and $A$ are true, then we can infer $B$ to be true, in the other three cases we cannot determine the truth value of $B$. Table 5 is the negative expression of hypothetical inference: if $A \leq^{-1} B$ is true and $\mathrm{B}$ is false, then we can infer $A$ to be false, in the other three cases we cannot determine the truth value of $A$.

Critics of mutually-inversistic logic might say: "Tables 3, 4, and 5 are not closed, the truth values participating the operations are $F$ and $T$, while the truth values yielding are $F, T, n$, and $u$ ". The answer of the author is: "The operations are closed, because the two indeterminacies cannot be generated: if it need not determine, then we do not determine; if it cannot determine, then we do not determine."

\subsection{Mutually inverse implication is free from implication paradoxes}

Material implication has implicational paradoxes, e.g., "if the Moon is made from green cheeses, then $2+2$ equals 4", $\mathrm{P} \wedge \neg \mathrm{P} \rightarrow \mathrm{Q}$, etc. Implicational paradoxes have two characteristics: (1) If the antecedent is false or the consequent is true, then the antecedent implies the consequent; (2) there can be no nexus of contents between the antecedent and the consequent. In mutually-inversistic logic it is required that (1) the antecedent not be permanently false, the consequent not be permanently true; (2) the antecedent and the consequent share the same variable. So, in mutually inverse implicational propositions, the two characteristics of implicational paradoxes are eliminated, and the propositions are free from implicational paradoxes. Take $\operatorname{man}(x) \leq^{-1} \operatorname{mortal}(x)$ as an example. Man(Aristotle) is true, so the antecedent is not permanently false. Mortal(stone) is false, so the consequent is not permanently true. Thus characteristic (1) of implicational paradoxes is eliminated. The antecedent and the consequent share the same variable $x$, thus characteristic (2) of implicational paradoxes is eliminated. So, $\operatorname{man}(x) \leq{ }^{-1} \operatorname{mortal}(x)$ is not an implicational paradox. By the way, in mutually-inversistic predicate calculus, quantifiers are not introduced, $x$ 's shared by both the antecedent and the consequent is relevantly bound. The idea comes from relevance logic. Of course, there are other kinds of boundness. But relevant boundness is the basic kind of boundness that all mutually inverse implication propositions have.

\section{Fuzzy logic}

In fuzzy logic, truth values are extended from $F(0)$ and $T(1)$ to the closed interval $[0,1]$. Fuzzy negation $\neg a$ is defined as $1-a$. Fuzzy conjunction $a \wedge b$ is defined as $\min (a, b)$. Fuzzy implication $a \rightarrow b$ is defined as $\max (1-a, b)$.

\section{Mutually-inversistic fuzzy logic}

In mutually-inversistic fuzzy logic, truth values are also extended from $F(0)$ and $T(1)$ to the closed interval [0, 1].

\subsection{Mutually inverse fuzzy negation $\neg_{\mathrm{f}}$}

Mutually inverse fuzzy negation $\neg_{\mathrm{f}}$ is defined as $\neg_{\mathrm{f}} a=$ def $1-a$, the same as fuzzy logic. In mutually-inversistic logic, there are $\neg F=T, \neg T=F$. In mutually-inversistic fuzzy logic, there are $\neg_{\mathrm{f}} 0=1, \neg_{\mathrm{f}} 1=0$. Therefore, $\neg$ is a special case of $\neg_{\mathrm{f}}$.

\subsection{Mutually inverse fuzzy conjunction $\wedge_{f}$}

Mutually inverse fuzzy conjunction $\wedge_{\mathrm{f}}$ is defined as

$a_{1} \wedge_{\mathrm{f}} a_{2}=\operatorname{def} \frac{a 1+a 2}{2}$

In mutually-inversistic logic, there are $F \wedge F=F$, $T \wedge T=T$. In mutually-inversistic fuzzy logic, there are $0 \wedge_{\mathrm{f}} 0=0,1 \wedge_{\mathrm{f}} 1=1$. These two cases of $\wedge$ are the special cases of $\wedge_{\mathrm{f}}$. In mutually-inversistic logic, there are $F \wedge T=F, T \wedge F=F$. While in mutually-inversistic fuzzy logic, there are $0 \wedge_{\mathrm{f}} 1=\frac{0+1}{2}=0.5,1 \wedge_{\mathrm{f}} 0=\frac{1+0}{2}=0.5$. These two cases of $\wedge$ are not the special cases of $\wedge_{\mathrm{f}}$.

The two conjuncts can have weights: 
$a_{1} \wedge_{\mathrm{f}} a_{2}=\operatorname{def} \frac{w 1 a 1+w 2 a 2}{w 1+w 2}$

\subsection{Mutually inverse fuzzy implication $\leq_{\mathrm{f}}^{-1}$}

"If $A(x)$ is $v$, then $C(x)$ is $w$ " where $\mathrm{v}$ and $\mathrm{w}$ are fuzzy concepts is a mutually inverse fuzzy implication proposition, denoted by $A \leq_{\mathrm{f}}^{-1} C$ where $A$ and $C$ are fuzzy sets. For example, "if $x$ study English every day longer, then $x$ 's English exam score is better" where longer and better are fuzzy concepts; is a mutually inverse fuzzy implication proposition, denoted by $T \leq_{\mathrm{f}}^{-1} S$ where $T$ and $S$ are fuzzy sets. $X$ in "if $A(x)$ is $\mathrm{v}$, then $C(x)$ is w" ranges over $\mathrm{n}$ instances $\left\{e_{1}, \ldots, e_{i}, \ldots, e_{n}\right\}$. The membership grade of $e_{i}$ to $A$ is $a_{i}$, to $C$ is $\mathrm{c}_{i} ; a_{i}, c_{i} \in[0,1]$ where 1 denotes absolute truth, 0 denotes absolute falsity. From $\mathrm{a}_{i}$ and $\mathrm{c}_{i}$, we can determine the strength of support $\mathrm{ss}_{i}$ of $\mathrm{e}_{i}$ to $A \leq_{\mathrm{f}}^{-1}$ $C, \mathrm{ss}_{i} \in[0,1]$ where 1 denotes absolute support, 0 denotes absolute opposition, 0.5 denotes neutral or "need not determine whether it is true or false". For example, $x$ in "if $x$ study English every day longer, then $x$ 's English exam score is better" ranges over the instances \{Alice, Bob, John, Max, Pat, Sam, Ted\}. Bob studies English 3 h every day. $5 \mathrm{~h}$ are regarded as long enough, $1 \mathrm{~h}$ is regarded as short enough, so, the membership grade of Bob's $3 \mathrm{~h}$ to $T$ is 0.5 . Bob scored 90 points in the English exam. In Chinese exam system, if one scored 60 points, then one passed the exam, and 100 points is the full mark. So, the membership grade of Bob's 90 points to $S$ is 0.75 . From the membership grades of 0.5 and 0.75 we can determine Bob's strength of support $\mathrm{ss}_{\mathrm{Bob}}$ to $T \leq_{\mathrm{f}}^{-1} S$ is 0.75 .

From $a_{i}$ and $c_{i}$ to determine $\mathrm{ss}_{i}$ is a binary function: $\mathrm{ss}_{i}=f\left(a_{i}, c_{i}\right)$. Just as we can use contour lines to depict a three-dimensional topography in a two-dimensional plane, we can use equi_strength_of_support lines to depict the three-dimensional function $\mathrm{ss}_{i}=f\left(a_{i}, c_{i}\right)$ in the two-dimensional plane. The diagram of equi_strength_of_support line is shown in Fig. 5.

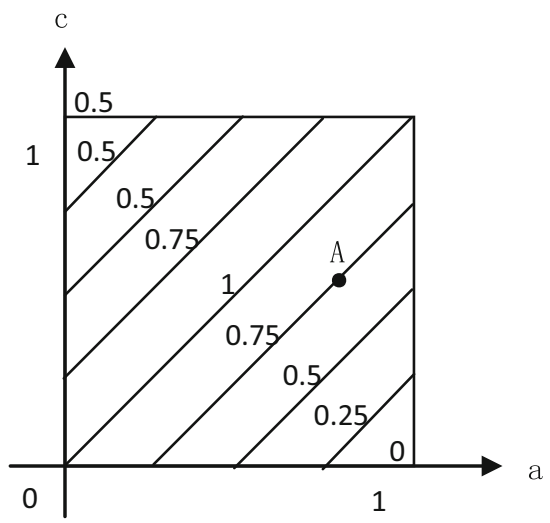

Fig. 5 Diagram of equi_strength_of_support line
There are infinitely many equi_strength_of_support lines in Fig. 5, we only draw a few of them. The first, second, third, and fourth row of Table 3 correspond to the bottom-left, top-left, bottom-right, and top-right corner of Fig. 5, therefore, the inductive composition of $\leq^{-1}$ is a special case of the inductive composition of $\leq_{\mathrm{f}}^{-1}$. According to Fig. 5, if we know $a$ and $c$ then we can determine ss. For example, suppose $a=0.75, c=0.5$, that is the point $A$ in Fig. 5, which is on the equi_strength_of_support line 0.75 , so, ss $=0.75$.

Every equi_strength_of_support line in Fig. 5 can be written as a slope-intercept equation:

$c=a+$ intc

From (3) we can obtain:

intc $=c-a$

Figure 5 depicts the relationship among $a, c$, and ss. But by (4), from $a$ and $c$ we can determine intc. So, according to Fig. 5, we can express the relationship between intc and ss as a piecewise function:

$\mathrm{ss}=\left\{\begin{array}{l}0.5, \quad 0.5 \leq \text { intc } \leq 1 \quad \text { (neutralarea) } \\ - \text { intc, } \quad 0 \leq \text { intc }<0.5 \quad \text { (supportarea) } \\ 1+\text { intc }, \quad-0.5<\text { intc }<0 \text { (supportarea) } \\ 1+\text { intc }, \quad \text { int } c=-0.5 \text { (neutralarea) } \\ 1+\text { intc }, \quad-1 \leq \text { intc }<-0.5 \text { (oppositionarea) }\end{array}\right.$

Suppose $a=0.75, \quad c=0.5, \quad$ then by (4), intc $=0.5-0.75=-0.25$. intc falls into the third row of (5), so, ss $=1+(-0.25)=0.75$.

After $\mathrm{ss}_{1}, \ldots, \mathrm{ss}_{i}, \ldots, \mathrm{ss}_{n}$ are all computed, then we can mine the membership grade ma of the mutually inverse fuzzy proposition $A \leq_{\mathrm{f}}^{-1} C$ :

$\mathrm{ma}=\left(\mathrm{ss}_{1}+\cdots+\mathrm{ss}_{\mathrm{i}}+\cdots+\mathrm{ss}_{n}\right) / n$

$A \leq_{\mathrm{f}}^{-1} C$ with its membership grade ma serve as the major premise, $A_{j}$ with its membership grade $\mathrm{a}_{j}$ serve as the minor premise, by fuzzy hypothetical inference, we can infer $C_{j}$ with its membership grade $\mathrm{a}_{j}$, ma is the possibility of $C_{j}$.

\section{Membership grade mining of mutually inverse fuzzy implication propositions}

Suppose we have an English study database shown in Table 6 . We want to mine the mutually inverse fuzzy implication proposition "if $x$ study English every day longer, and $x^{\prime}$ 's attention is focused, then $x$ 's English exam score is better" denoted by $\frac{w 1 T+w 2 A}{w 1+w 2} \leq{ }_{f}^{-1} S$. Longer, focused, and better are fuzzy concepts, their membership functions are shown in Figs. 6, 7, and 8. 
Table 6 An English study database

\begin{tabular}{llcc}
\hline Universe $U$ & $\begin{array}{l}\text { English study time } \\
\text { every day } T(\mathrm{~h})\end{array}$ & $\begin{array}{l}\text { Attention } \\
A(\%)\end{array}$ & $\begin{array}{l}\text { English exam } \\
\text { score } S \text { (points) }\end{array}$ \\
\hline Alice & 2 & 25 & 70 \\
Bob & 3 & 100 & 90 \\
John & 3 & 75 & 80 \\
Max & 4 & 75 & 100 \\
Pat & 2 & 25 & 70 \\
Sam & 3 & 0 & 70 \\
Ted & 5 & 100 & 50 \\
\hline
\end{tabular}

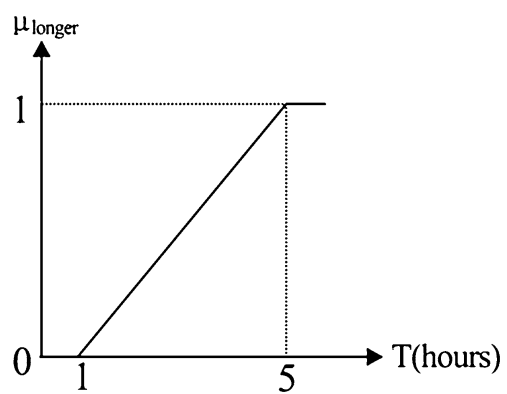

Fig. 6 Membership function for longer

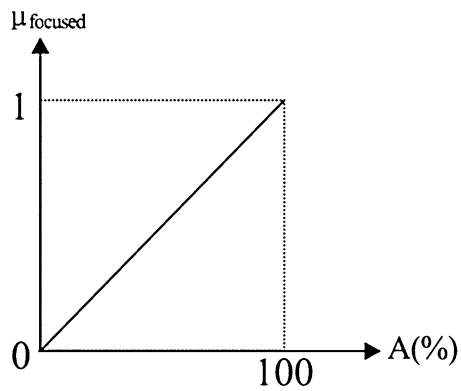

Fig. 7 Membership function for focused

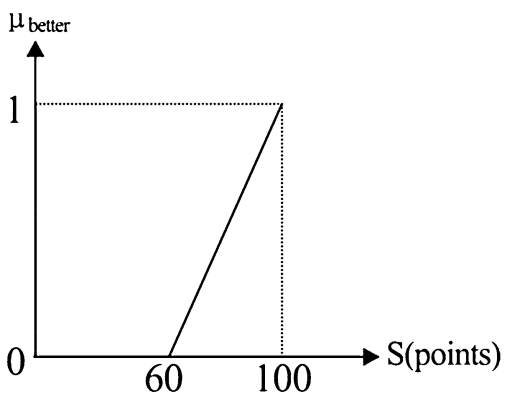

Fig. 8 Membership function for better

The membership grade mining of $\frac{w 1 T+w 2 A}{w 1+w 2} \leq_{f}^{-1} S$ is divided into two steps. Step 1 is to determine the weights $w_{1}$ and $w_{2}$. Step 2 is to determine the membership grade of $\frac{w 1 T+w 2 A}{w 1+w 2} \leq_{\mathrm{f}}^{-1} S$.

\subsection{Step 1: determining the weights}

We use the first four instances of Table 6 to determine the weights. For Alice, $T$ (Alice) $=2 \mathrm{~h}$, according to Fig. 6, $a_{11}=0.25$. A(Alice) $=25 \%, \quad$ according to Fig. 7, $a_{12}=0.25$. $S$ (Alice) $=70$ points, according to Fig. 8, $c_{1}=0.25$. Likewise, for Bob, $a_{21}=0.5, a_{22}=1$, $c_{2}=0.75$. The first simultaneous equations become

$\left\{\begin{array}{l}w_{11} \times 0.25+w_{12} \times 0.25=0.25 \\ w_{11} \times 0.5+w_{12} \times 1=0.75\end{array}\right.$

Solving (7), we obtain $w_{11}=0.5, w_{12}=0.5$.

For John, $a_{31}=0.5, a_{32}=0.75, c_{3}=0.5$. For Max, $a_{41}=0.75, a_{42}=0.75, c_{4}=1$. The second simultaneous equations become

$\left\{\begin{array}{l}w_{21} \times 0.5+w_{22} \times 0.75=0.5 \\ w_{21} \times 0.75+w_{22} \times 0.75=1\end{array}\right.$

Solving (8), we obtain $w_{21}=2, w_{22}=-2 / 3$.

$w_{1}=w_{11}+w_{21}=2.5, \quad w_{2}=w_{12}+w_{22}=-0.17$. And the mutually inverse fuzzy implication proposition becomes

$\frac{2.5 T-0.17 A}{2.33} \leq_{\mathrm{f}}^{-1} S$

\subsection{Step 2: determining the membership grade of $\frac{2.5 T-0.17 A}{2.33} \leq_{\mathrm{f}}^{-1} S$}

The second step is, for the remaining instances of Table 6, to determine the strength of support $\mathrm{ss}_{i}$ (using (5)) and then the membership grade ma (using (6)) of $\frac{2.5 T-0.17 A}{2.33} \leq_{\mathrm{f}}^{-1} S$.

For Pat, $a_{51}=0.25, a_{52}=0.25 . a_{5}=\frac{2.5 \times a 51-0.17 \times a 52}{2.33}=$ 0.25. $c_{5}=0.25$. intc $c_{5}=c_{5}-a_{5}=0$. According to the second row of (5), $\mathrm{ss}_{5}=1-$ intc $_{5}=1$. For Sam, $a_{61}=0.5, \quad a_{62}=0, \quad \mathrm{a}_{6}=0.536, \quad c_{6}=0.25 . \quad$ intc $_{6}=-$ $c_{6}-a_{6}=-0.286$. According to the third row of (5), $\mathrm{ss}_{6}=1+$ intc $_{6}=0.714 . \quad$ For Ted, $a_{71}=1, \quad a_{72}=1$. $a_{7}=1 . c_{7}=0$. Int $c_{7}=-1$. According to the fifth row of (5), $\mathrm{ss}_{7}=1+\operatorname{int} c_{7}=0$. From the fifth row of (5) we know that Ted is a counterexample. Both the counterexamples and the neutral examples are exceptional examples and are not taken into account of the membership grade ma, but are outputted separately.

$\mathrm{Ma}=\left(\mathrm{ss}_{5}+\mathrm{ss}_{6}\right) / 2=0.857$. Thus, the membership grade of $\frac{2.5 T-0.17 A}{2.33} \leq_{\mathrm{f}}^{-1} S$ is 0.857 .

By the way, the order of the instances is not important.

\subsection{Fuzzy hypothetical inference}

The aim of mining the membership grade ma of $\frac{2.5 T-0.17 A}{2.33} \leq_{\mathrm{f}}^{-1} S$ is to use it as the major premise to make 
fuzzy hypothetical inference. Suppose Dick studies English $2 \mathrm{~h}$ every day, and Dick's attention is $25 \%$. Then $T$ (Dick) $=2 \mathrm{~h}, \quad$ according $\quad$ to Fig. $6, \quad a_{81}=0.25$. $A($ Dick $)=25 \%, \quad$ according to Fig. 7, $a_{82}=0.25$. $a_{8}=\frac{2.5 T-0.17 A}{2.33}=0.25$. By fuzzy hypothetical inference, we can infer $c_{8}=0.25$. According to Fig. 8, we convert $c_{8}$ back to points, $S$ (Dick) $=60+40 \times 0.25=70$ points. $\mathrm{Ma}=0.857$ is the possibility of Dick's English exam score being 70 points, that is, Dick's English exam score is predicted to be 70 points with possibility $85.7 \%$.

\section{Comparison of mutually-inversistic fuzzy logic with Zadeh's fuzzy logic}

The following are the differences between mutually-inversistic fuzzy logic and Zadeh's fuzzy logic:

1. In mutually-inversistic fuzzy logic, for the fuzzy set " $x$ 's English exam score is better" there is only one membership function Fig. 8. Even if the membership grade is very low, e.g. in Sect. $6.3 c_{8}=0.25$, it still use Fig. 8. This means that only one mutually inverse fuzzy implication proposition $T \wedge_{\mathrm{f}} A \leq_{\mathrm{f}}^{-1} S$ is needed. The same is true for Fig. 6 and Fig. 7. While corresponding to Fig. 8, in the conventional fuzzy association rule mining (Subramanyam and Goswami 2006) there are three membership functions. For better score, there is Fig. 9, for plain score, there is Fig. 10,

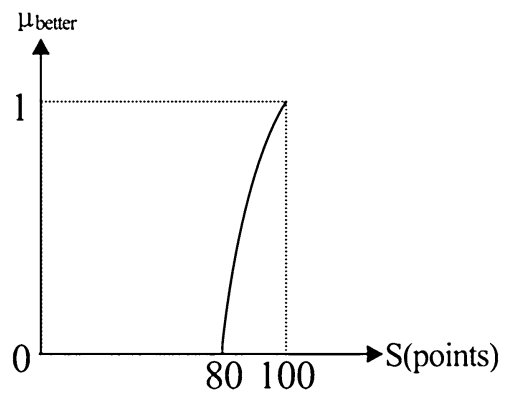

Fig. 9 Membership function for better

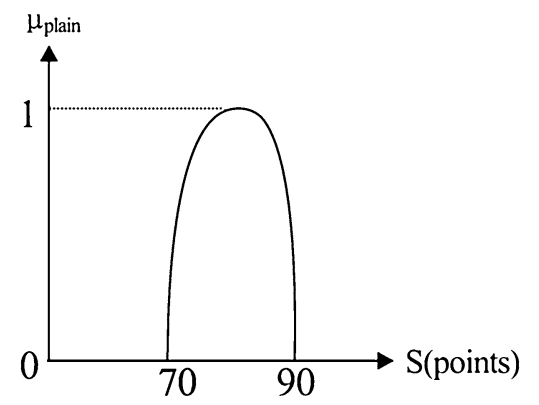

Fig. 10 Membership function for plain

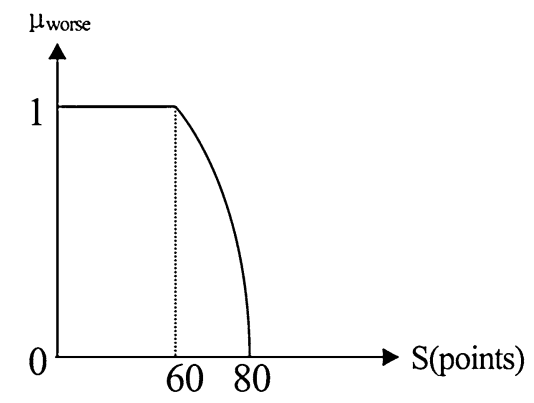

Fig. 11 Membership function for worse

for worse score, there is Fig. 11. In this example, we even need five membership functions, one for very good score, one for good score, one for plain score, one for bad score, one for very bad score. Therefore, in the conventional fuzzy association rule mining, nine fuzzy implication propositions are needed. "If $x$ study English every day longer, and $x$ 's attention is more focused, then $x$ 's English exam score is very good"; "if $x$ study English every day longer, and $x$ 's attention is medium, then $x$ 's English exam score is good"; "if $x$ study English every day longer, and $x$ 's attention is less focused, then $x$ 's English exam score is plain"; ..., "if $x$ study English every day shorter, and $x$ 's attention is less focused, then $x$ 's English exam score is very bad". Mutually-inversistic fuzzy logic reduce the nine rules into one. This is one of the advantages of mutually-inversistic fuzzy logic over the conventional fuzzy association rule minging.

2. A membership function may not be linear, any monotonic function will do. But the linear functions such as Figs. 6, 7, and 8 have advantages: they can be constructed objectively. Take Fig. 8 as an example. In Chinese exam system, if one does not score 60 points, then one fails the exam; if one scores 100 points, then one gets full mark. So, 60 points is the worst score (membership grade 0), 100 points is the best score (membership grade 1). Draw a straight line between $(60,0)$ and $(100,1)$, we obtain the membership function of better, i.e., Figure 8. While in the conventional fuzzy association rule mining, the membership functions such as Figs. 9, 10, and 11 are constructed subjectively.

\section{Concluding remarks}

Mutually inverse implication inherits from and makes several improvements to material implication. Mutually inverse fuzzy implication combines mutually inverse implication and fuzzy logic. The membership grade mining 
algorithm of mutually inverse fuzzy implication propositions is given, which comprises two steps. The first step is to determine the weights of the two antecedents. The second step is to mine the membership grade of the mutually inverse fuzzy implication proposition. The aim of mining the membership grade of the mutually inverse fuzzy implication proposition is to use it as the major premise to make fuzzy hypothetical inference.

\section{References}

Anderson AR, Jr ND (1975) Belnap, entailment: the logic of relevance and necessity, vol 1. Princeton University Press, Princeton
Emrouznejad A, Marra M (2014) Ordered weighted averaging operators 1988-2014: a citation-based literature survey. Int J Intell Syst 29:994-1014

Hamilton AG (1978) Logic for mathematicians (Revised edition). Cambridge University Press, Cambridge

Kuok CM, Fu AWC, Wong MH (1997) Mining fuzzy association rules in database. In: Proceedings of the ACM Sixth International Conference on Information and Knowledge Management, Las Vegas, Nevada, USA, 10-14, 1997

Subramanyam RBV, Goswami A (2006) Mining fuzzy quantitative association rules. Exp Syst 23(4):212-225

Yager RR (1988) On ordered weighted averaging aggregation operators in multi-criteria decision making. IEEE Trans Syst Man Cybern 18:183-190

Zadeh LA (1965) Fuzzy sets. Inf Control 8(3):338-353

Zhou X (2013) Mutually-inversistic logic, mathematics, and their applications. Central Compilation and Translation Press, Beijing 